This item was submitted to Loughborough's Research Repository by the author.

Items in Figshare are protected by copyright, with all rights reserved, unless otherwise indicated.

\title{
Factors affecting the level of success of community information systems
}

PLEASE CITE THE PUBLISHED VERSION

PUBLISHER

(C) Emerald

LICENCE

CC BY-NC-ND 4.0

\section{REPOSITORY RECORD}

Coombs, Crispin, Neil Doherty, and John Loan-Clarke. 2019. "Factors Affecting the Level of Success of Community Information Systems". figshare. https://hdl.handle.net/2134/1133. 
This item was submitted to Loughborough's Institutional Repository by the author and is made available under the following Creative Commons Licence conditions.

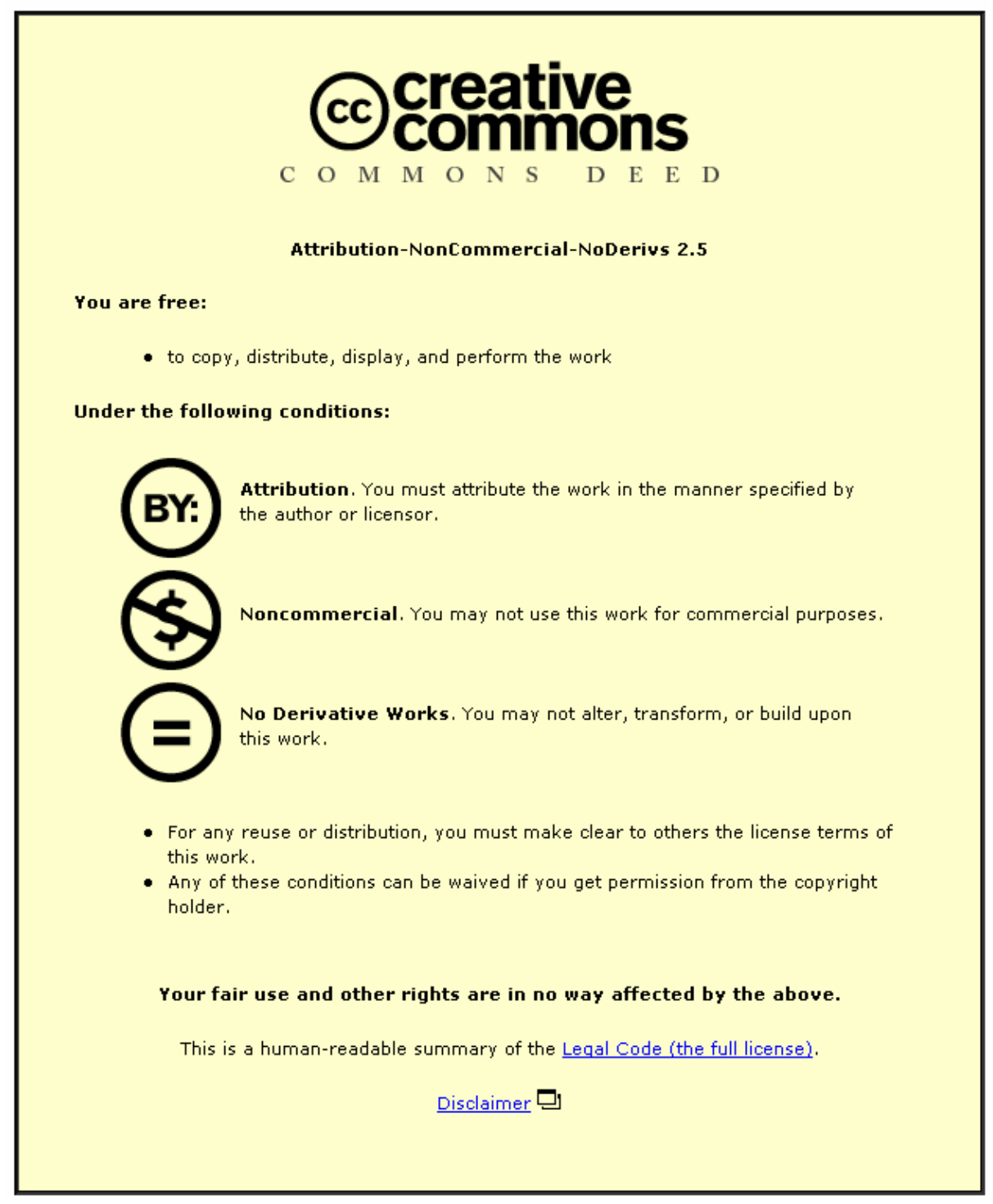

For the full text of this licence, please go to: http://creativecommons.org/licenses/by-nc-nd/2.5/ 


\title{
L Loughborough University
}

\section{Factors Affecting the Level of Success of Community Information Systems}

\author{
Authors: \\ Coombs, C. R., Doherty, N. F., \& Loan-Clarke, J. \\ The Business School, Loughborough University
}

Autobiographical note:

Crispin Coombs, Doctoral Research Student, The Business School, Loughborough University c.r.coombs@lboro.ac.uk

Neil Doherty, Lecturer in Management Information Systems, The Business School, Loughborough University, n.f.doherty@lboro.ac.uk

John Loan-Clarke, Lecturer of Human Resource Management, The Business School, Loughborough University, j.loan-clarke@lboro.ac.uk

Mailing Address:

Dr Neil Doherty,

The Business School,

Loughborough University,

Loughborough, LE11 3TU,

United Kingdom.

Telephone: 01509223128

Email: N.F.Doherty@lboro.ac.uk

Fax: $\quad 01509223960$ 


\section{Factors Affecting the Level of Success of \\ Community Information Systems}

The factors that influence the ultimate level of success or failure of systems development projects have received considerable attention in the academic literature. However, previous research has rarely targeted different instances of a common type of system within a homogenous organisational sector. This paper presents the results of a survey of IM\&T Managers within Community Trusts to gain insights into the factors affecting the success of Community Information Systems. The results demonstrate that the most successful operational systems were thoroughly tested prior to implementation and enjoyed high levels of user and senior management commitment. Furthermore, it has been shown that there is a relationship between the level of organisational impact and systems success, with the most successful systems engendering changes to the host organisation's culture, level of empowerment and clinical working practices In addition to being of academic interest, this research provides many important insights for practising IM\&T managers.

Key Words: Systems development; Success; Failure; NHS; Community Information Systems; United Kingdom 


\section{$\underline{\text { INTRODUCTION }}$}

In both the public and private sectors, the acquisition, and successful introduction of information technology is by no means a straightforward process. Large numbers of information systems projects are either excessively over budget, months or years behind schedule, of poor quality, or simply fail to adequately satisfy users' requirements. Statistics on the success and failure of information systems are plentiful, and generally depressing (For example: Lyytinen \& Hirschheim, 1987; Kearney, 1990; Hochstrasser \& Griffiths, 1991; Clegg et al, 1997).

One sector which has enjoyed high levels of investment in information technology, yet failed to fully reap its benefits, is the National Health Service (NHS) in the United Kingdom (N.A.O., 1991). The recognised importance of IT within the NHS stems from the mid 1980s, with the publication of the inaugural national strategy for IT (DHSS, 1986). Since then there has been a headlong drive for improvements in the quantity and quality of information, resulting in millions of pounds being invested in IT (Keen, 1994). In 1991, however, a National Audit Office report (N.A.O., 1991) concluded that: 'The management of computer systems [within the NHS] was often weak, with many failures to follow good practice, resulting in poor value for money'. In order to arrive at these disconcerting conclusions, the same report had assessed eleven NHS computer projects on five key features of best practice, and had found that the major shortcomings included: 'incomplete feasibility studies; loose contractual arrangements; inadequate planning; weak control and an absence of postimplementation reviews'. More recently, this appraisal of the situation has been supported by Clegg et al, (1997) who conclude: 'The health sector is still seen as performing rather poorly in the field of IT'. 
The focus of this paper is the development, implementation and impact of information systems within community health services. In 1990 the white paper 'Working for Patients' highlighted the need for 'all community health services providers to have computerised information systems as soon as practicable and for existing systems implementations to be completed and made to work well' (Information Management Group, 1990). More recently, an Audit Commission report (Audit Commission, 1997) stressed the need for such community information systems (CIS) to be patient-based to support in clinical decisionmaking, as well as supporting administrative and contract management activities. Unfortunately, the same report (Audit Commission, 1997) noted the 'ineffectiveness of information systems' within this sector. More specifically, it noted that: 'most information systems provide only limited support to front-line staff'; 'many systems are out-dated and badly designed' and 'the introduction of technology is usually badly planned'. In a similar vein, the recent NHS IM\&T Strategy (Burns, 1998) also identifies failings in existing community information systems stating that 'the inadequacies of information systems to support community health staff have been apparent for many years'.

Whilst the performance of information systems within Community Trusts has been assessed by the Audit Commission, it has not, as yet, been reviewed through empirical, academic research. The aim, therefore, of this piece of research is to redress the balance by critically evaluating the success of Community Information Systems (CIS) in the context of current knowledge about success and failure in systems development and implementation. Therefore, the structure of this paper is: firstly, a brief overview of the relevant information systems literature; secondly, a statement of the research objectives for this project; thirdly, a description of the methods by which the research instrument was developed, validated and ultimately executed; fourthly, the research results are presented and discussed; finally, their 
importance is assessed in the concluding section.

\section{CONTEXTUAL BACKGROUND AND RESEARCH OBJECTIVES}

In the past twenty years much interest has been generated in the identification of factors critical to the successful outcome of systems development projects. A range of empirical and in-depth studies have been conducted which examine success factors in the development and implementation of information systems (For example: Rademacher, 1989; Cerullo, 1980; Yap et al, 1992; Sauer, 1993; Willcocks \& Margetts, 1994; Whyte \& Bytheway, 1996; Flowers, 1997; Li, 1997). These, and other studies, have helped to focus IT professionals' attention on the importance of factors such as: user involvement (Wong \& Tate, 1994; Whyte \& Bytheway, 1996); senior management commitment (Cerullo, 1980; Sauer, 1993); Staff training (Miller \& Doyle, 1987; Whyte \& Bytheway, 1996) and systems testing (Ennals, 1995; Flowers, 1997). Whilst all these studies have helped to develop a formidable body of knowledge with regard to 'best practice' in systems development, little research has been conducted into the application of best practice, in systems development projects, within the community sector of the NHS.

A further important strand of research concerns the organisational impact of information systems. It has been recognised that the level of penetration and sophistication of information technology is growing rapidly, and with this expansion goes a concomitant increase in the level of the organisational impact of information technology. For example, it has been found that the implementation of information systems can precipitate changes in: organisational structure (Markus \& Robey, 1983; Stebbins et al, 1995); organisational culture (Bufferfield \& Pendegraft, 1996; Pliskin et al, 1993); working practices (Eason, 1988; Hornby et al, 1992) and the distribution of power (Sauer, 1993; Thach \& Woodman, 1994). Recent research 
(Doherty \& King, 1998) also suggests that the organisational impact of systems is gradually increasing. Venkatraman (1991) suggests that there is a direct relationship between the level of organisational impact and the resultant level of organisational benefit; the higher the impact, the greater the potential benefit. To date, little empirical work has been conducted to explore this relationship, in either the NHS or more generally within other organisational contexts.

To overcome these weaknesses, an empirical research project, was initiated, targeting a single organisational sector, in which a standard application of IT has been developed over a long time period and is still currently being implemented. This approach ensured that the following research objectives could be addressed:

1. To explore the relationship between the ability of a CIS project team to adopt best practice, and the resultant level of success or failure of the operational information system.

2. To explore the relationship between the level of organisational impact engendered by the system, and the resultant level of success or failure of the operational information system.

It was envisaged that through the exploration of these issues it would be possible to provide advice to the NHS in general, and Community Trusts in particular, with regard to the successful development and implementation of information systems.

\section{RESEARCH METHODS}

In order to study the factors affecting the success and failure of community information 
systems (CIS) a two phase approach was adopted combining qualitative and quantitative research methods. It has been argued that combining these methods in IS research can prove useful in building a wider picture of the phenomenon studied (Reichardt \& Cook, 1989), can enable the validation of findings (Jick, 1979) and can help in explaining diverging results (Trend, 1989). This research project used a combination of qualitative methods, in the initial exploratory stage, and a quantitative research instrument, in the subsequent data collection phase (Miles \& Huberman, 1994). The first phase of the research project employed a series of semi-structured interviews designed to investigate the success and failure factors identified in the IS literature in the context of the community health sector. The second phase of the research project involved a questionnaire survey of all Community Care Trusts in England and Wales. Whilst this paper focuses predominately on the quantitative results of the research project, as these findings are more appropriate for making generalisations, the main results from the interviews are also briefly summarised.

\section{Qualitative Research Method and Results}

Thirteen semi-structured interviews were conducted with key stake holders at Central Nottinghamshire Healthcare (NHS) Trust (CNHT), as this organisation has a research link with the Business School at Loughborough University. The interviewees ranged from the chief executive to community service managers and clinical staff. The results from the interviews confirmed the importance of the issues identified from the literature and identified a small number of additional aspects that may be important in CIS projects. The main factors identified as being important to the success of a CIS were: good quality education, training and support; user involvement throughout the project; and senior management commitment. In terms of the organisational impact of the CIS, changes were noted in the organisational culture, some of the nurse's working practices, and users generally being 
empowered with greater information. In addition to being of interest in their own right, these results were also a great help in establishing the focus and content of the quantitative research instrument.

\section{Quantitative Research Method}

A draft questionnaire was developed which was based upon issues identified from the literature and issues highlighted from the qualitative research phase of the project. The research instrument was thoroughly pre-tested by a selection of appropriate academics, staff at CNHT, I,M \& T Managers from five other Community Trusts and members of the NHS Executive Information Management Group. When possible a personal interview pre-test approach was used, the questionnaire being completed and its content and ease of use discussed (Churchill, 1988). Alternatively, the draft questionnaire was returned with written comments and suggestions. On the whole the pre-testers were happy with the content of the questionnaire although a number of interesting enhancements were suggested and ultimately adopted.

The final draft questionnaire was broken down into three main sections each of which is briefly reviewed below:

\section{Adoption of best practice}

This section was concerned with measuring the extent to which Community Trusts had been successful in the adoption of best practice. The questions covered a range of areas including: testing of the system; education, training and support; user involvement; and top management support. The questions were primarily derived from the NHS's own list of 'best practice' factors (N.A.O., 1996), although other literature sources (For example: Cerullo, 1980; Whyte 
\& Bytheway, 1996; Flowers, 1997) and the results of the qualitative research were used to validate and focus them.

\section{The level of organisational impact}

The second section of the questionnaire investigated the degree of impact that the CIS had on the organisation. These areas included the influence of the CIS on: levels of empowerment; organisational culture and working practices. These questions were primarily derived from the literature (For example: Doherty \& King 1998, Clegg et al, 1997) and from issues highlighted in the qualitative research.

\section{Performance of the system}

The final section of the questionnaire was designed to evaluate the system's success, based upon a range of distinct, yet complementary, measures. The ten success measures, such as 'user satisfaction', ‘systems accuracy / reliability’, and ‘information quality’, adopted were strongly influenced by the taxonomy of success measures identified by DeLone and Maclean (1992).

Each of the three sections were operationalised by asking respondents to indicate the extent to which they agreed or disagreed with each statement on a scale of 1-5 where 1 was strongly disagree and 5 was strongly agree. In addition an 'other comments' section was provided to give respondents the opportunity to qualify their answers, and a background section requested information about the respondent's Trust and their CIS.

The questionnaire was targeted at the IM\&T managers in all community, mental health and learning disability NHS trusts in England and Wales. It was envisaged that this selection 
strategy would ensure that all community healthcare trusts were incorporated in the sampling frame. A database was created from the 1995/96 and 1997/98 NHS Yearbooks whilst additional information was provided by the NHS Management Executive. All the questionnaires were sent to named addressees and in cases where no IM\&T manager was identified the Chief Executive was used as an alternative. The database had 236 potential respondents and that was considered to be the total population of community, mental health and learning disability Trusts in England and Wales.

The final draft of the questionnaire was piloted on a $10 \%$ sample of the main survey population. Twenty questionnaires were sent out and 12 were returned giving a very encouraging response rate of $60 \%$. Analysis of the responses indicated no problems with the content or structure of the questionnaire and no alterations were made. Including the pilot, a total of 136 responses were received giving a very high response rate of 58\%. Although the survey guaranteed confidentiality, respondents were offered the opportunity to receive a copy of the results if they were interested. It was encouraging that over $80 \%$ of the respondents requested a copy of the results, giving some indication of the high interest in this topic within the community health sector.

\section{RESEARCH RESULTS}

Of the 136 responding trusts, ninety two (68\%) respondents indicated that their Trust had a community information system. Of these, one had not yet started implementation, 49 (36\%) had partially implemented and 42 (31\%) had fully implemented their system. Of the remaining respondents, only 10 (7\%) stated that their trust had no intention of acquiring a CIS in the next 24 months. This result clearly shows that the uptake and application of CISs is well underway throughout England and Wales and that their use is becoming increasingly 
important in the delivery of healthcare in the community.

A summary of the scores for each of the ten success measures is presented in Table I. These results suggest that the performance of community information systems has, to date, been modest with all the average success scores clustered around the midpoint of three on the Likert scales. However, it is interesting to note that whilst the impact of CIS on direct patient care is perceived to have been limited, its positive contribution to managerial decisionmaking is readily acknowledged.

Take in Table I here

In order to explore the two research objectives identified at the end of section two of this paper, it was necessary to generate an overall success score for each responding trust. This overall success score was derived by averaging the ten individual success measures. Having confirmed the statistical validity (Hair et al, 1995) of combining the ten individual measures of success, average success scores were derived for each of the 75 respondents who had provided a complete set of success scores. The remainder of this section uses the summary success score to explicitly explore the two research objectives

\section{The Adoption of Best Practice and the Resultant level of Success}

The relationship between each best practice factor and the overall success measure was explored by generating a series of correlation coefficients, utilising 2-tailed tests; the results are presented in Table II. These results indicate that 4 of the best practice factors have a relationship with the success score that is statistically significant at the 0.01 level. The highest of the coefficients was for extensive testing of the system and success. The 
importance of testing is recognised as a best practice factor by the NHS (N.A.O., 1996) as well as the general IS literature (Ennals, 1995; Flowers, 1997) and therefore its significant score in this analysis simply supports existing theory.

Take in Table II here

Other key areas that also have significant correlations with success, at the $0.01 \%$ level, are providing support and help for staff during implementation, ensuring adequate user involvement and maintaining support for the project from top management. Further evidence of the importance of these factors was provided by the number of respondents who chose to make reference to them in the 'other comments' section of the questionnaire. For example, a representative selection of respondents' comments included: 'the inclusion of clinicians and IT specialists in a cohesive team has been invaluable'; 'successful implementation relies totally on user involvement and senior management commitment throughout the project', and 'training, ownership and support are key to a successful implementation'. These results confirm the findings of previous studies and reports (Audit Commission, 1997; Cerullo, 1980; Whyte \& Bytheway, 1996; Miller \& Doyle, 1987; N.A.O., 1996) as they suggest that those Trusts that deploy appropriate support and secure high levels of user participation and senior management commitment are likely to achieve higher levels of system’s success.

The two correlations that are significant at the $0.05 \%$ level both concern different aspects of training, namely the importance of designing broad training programmes and the need to allow for variations in the skill of users. These findings support previous studies that have indicated the importance of training in the successful introduction of information systems (Whyte \& Bytheway, 1996; Miller \& Doyle, 1987). 
It should be noted that while all these significant correlations do not indicate causality, they do provide evidence that these are positive steps that have been adopted in the development and implementation of successful community information systems. They also provide evidence that the best practice factors associated with the successful introduction of information technology within Community Trusts are consistent with existing information systems theory.

\section{The System's Organisational Impact and the Resultant level of Success}

The same correlation analysis approach used in the previous section was also used to study the relationship between system success and organisational impact. The results presented in Table III provide evidence to support the view that the level of organisational impact that an information system has within a Trust can influence the success of the system. More specifically, two positive correlations and two negative correlations were found to be statistically significant at the 0.01 level.

Take in Table III here

Positive correlations were identified for the system empowering the users and for the system modifying the organisational culture. The importance of empowerment was also highlighted in the initial qualitative phase of the research where one of the interviewees commented that: 'the only way to ensure that the planned benefits are ultimately realised is through the empowerment of users'. These findings, which support the work of Walton (1989), demonstrate the importance of explicitly considering these issues during the development and implementation process to ensure that the cultural and behavioural impacts of an information system are at least acceptable, if not positive. The positive relationship between clinical 
working practices and success, at the $0.05 \%$ level, is also interesting as it suggests that the impact of Community Information Systems is being felt outside the realms of management and administration. This finding provides some evidence that those Trusts that have heeded the Audit Commission's (1997) call for CIS to be 'patient-based to support in clinical decision-making' perceive their systems to be successful.

In terms of the significant negative correlations between the statements and success, the first can be viewed as a positive correlation as the statement itself is negative. The statement that the CIS has not improved the flow of information within the Trust has a negative correlation of -0.3777 that implies that there is a positive association between the CIS improving the flow of information within the Trust and the perceived success of the system. The only true negative correlation is between staff still keeping paper records to the same extent that they did prior to the CIS and the success score. The significant negative correlation of -0.5576 indicates that there is an expectation that the CIS should reduce the amount of paperwork that staff have to deal with if it is to be viewed as a success. If this does not occur then a duplication of work is likely to continue that will lead to dissatisfaction with the system from staff.

Previous research (Pliskin et al, 1993; Cooper 1994) has indicated that IT-induced organisational change can result in user resistance and ultimately system rejection. By contrast, these findings tend to support Venkatraman's (1991) proposition that there is a positive relationship between organisational impact and system's success. Indeed, it can be argued that those trusts that adopt a more proactive approach to organisational change and look to develop opportunities rather than attempting to maintain the status quo that existed before the information system are more likely to have a successful system. The lack of any 
significant correlation between changes in non-clinical working practices, organisational structure and processes, and success, however, suggests that these changes are likely to be more subtle in the way staff conduct their day-to-day activities rather than revolutionising their current working practices.

\section{CONCLUSIONS}

This paper presents a quantitative study in an important area of research which should be of interest to both IT professionals within the healthcare sector, as well as the IS community in general. The results of this research are important because they are based upon an exceptionally good response rate (58\%), of a survey targeted at a complete population, rather than simply a sample, namely the IT Managers of all Community Trusts within the UK.

When exploring the relationship between best practice and systems success, the research approach differed from the majority of previous studies by targeting different instances of a common type of system within a homogenous organisational sector. The survey has identified the key elements that comprise best practice with regards to systems development and implementation within the community sector and provides important insights to IT professionals about where they need to concentrate their efforts. Whilst these findings support the work of other information systems researchers, it is clear that many Trusts within the community sector, have as yet been unable to successfully adopt best practice.

The finding that there is a relationship between a system's organisational impact and its level of effectiveness should also be of great interest to IT practitioners in Community Trusts. It demonstrates that organisational change should be explicitly addressed during the development process and provides important insights into those areas that need to be 
concentrated upon if the information system is to be successful. This result also has wider interest to the IT community in general as there has been little empirical research into the relationship between organisational impact and systems’ success.

It is encouraging to see that the current NHS IM\&T Strategy supports a number of the key findings of this research, both in terms of best practice and organisational impact, which are explicitly identified as areas to be addressed in future systems development projects. For example, the strategy (Burns, 1998) highlights the importance of active stakeholder involvement in systems development projects, suggests that training strategies need to focus on the long-term development of an information culture and highlights the need to empower clinicians to use information technology to review and improve their clinical working practices. However, despite the positive steps that the NHS IM\&T Strategy is making its still remains to be seen whether the lessons it recommends will be translated into far higher levels of success than have been identified in this research. It is clear that if the NHS is going to achieve the goals that have been set in the new strategy it will have to radically improve on its past performance in the use of information and information technology.

Whilst this study has provided important insights into the strength of the relationships between systems success and the adoption of best practice and the system's organisational impact, further research is required to study the exact nature and implications of these relationships. Consequently, a further phase of this project has already been initiated, at a number of case study sites, to provide an in-depth analysis of the development, organisational impact and contribution of community information systems. Moreover, this follow-up study is targeting a wide range of managerial and clinical staff, in addition to IM\&T personnel, to investigate whether there are any significant variations in the perceptions of different 
stakeholder groups. It is envisaged that this study will result in the development of a set of practical prescriptions and approaches to aid IT practitioners in the development of successful systems.

\section{$\underline{\text { Acknowledgements }}$}

The authors would like to thank all of the members of Central Nottinghamshire Healthcare (NHS) Trust who gave up so much of their valuable time to participate in the early stages of this research, in addition to the large numbers of IM \& T Managers who responded to the survey. 


\section{REFERENCES}

Audit Commission, (1997) A Study of Information Management in Community Trusts, Audit Commission for Local Authorities and the National Health Service in England and Wales, London.

Bufferfield, J. and Pendegraft, N. (1996) "Cultural Analysis in IS Planning and Management”, Journal of Systems Management, Vol. 47 No. 2 pp14-18.

Burns, F. (1998) Information for Health. NHS Executive, Leeds.

Cerullo, M. J. (1980) "Information Systems Success Factors", Journal of Systems Management, Vol. 31 No. 12, pp. 10-19.

Churchill G. A., (1988) Basic Marketing Research, The Dryden Press, London.

Clegg, C., Axtell, C., Damadoran, L., Farbey, B., Hull, R., Lloyd-Jones, R., Nicholls, J. Sell, R. \& Tomlinson, C. (1997) "Information Technology: a study of performance and the role of human and organisational factors”, Ergonomics, Vol. 40, No. 9, pp. 851-871.

Cooper, R. B. (1994) “The inertial impact of culture on IT implementation”, Information \& Management, Vol. 27, pp. 17-31.

DeLone, W. H., and McLean, E. R. (1992) "Information Systems Success: The Quest for the Dependent Variable”, Information Systems Research, Vol. 3, No. 1, pp. 60-95.

DHSS, (1986) A National Strategic Framework for Information Management in the Hospital and Community Health Services, DHSS, London.

Doherty, N. F. \& King, M., (1998) “The consideration of organizational issues during the systems development process: an empirical analysis”, Behaviour \& Information Technology, Vol. 17, No. 1, pp. 41-51.

Eason, K., (1988) Information Technology and Organisational Change, Taylor \& Francis, London.

Ennals, R., (1995) Preventing Information Technology Disasters, Springer, London.

Flowers, S., (1997) “Towards predicting information systems failure”, In Avison, D. (Ed.) Key Issues in Information Systems, McGraw-Hill, Maidenhead, pp. 215-228.

Hair, J. F., Anderson, R. E., Tatham, R. L., and Black, W. C. (1995) Multivariate Data Analysis with Readings (Fourth Ed.), Prentice Hall, Engelwood Cliffs, New Jersey.

Hochstrasser, B \& Griffiths, C., (1991) Controlling IT Investment., Chapman \& Hall, London. 
Hornby, C., Clegg, C., Robson, J., McClaren, C., Richardson, S. \& O’Brien, P., (1992) “Human \& Organisational Issues in Information Systems Development”, Behaviour \& Information Technology, Vol. 11, No. 3, pp. 160-174.

Information Management Group, (1990) Working For Patients, Framework for Information Systems: The Next Steps, HMSO, London.

Jick, T. D. (1979) “Mixing qualitative and quantitative methods: triangulation in action”, Administrative Science Quarterly, Vol. 24, pp 602-611

Kearney, A. T., (1990) Barriers to the Successful Application of Information Technology, DTI \& CIMA, London.

Keen, J., (1994) Information Management in Health Services, The Open University Press, Buckingham.

Li, E. Y., (1997) "Perceived importance of information systems success factors: A meta analysis of group differences”, Information \& Management, Vol. 32, No. 1, pp. 1528.

Lyytinen, K. and Hirschheim, R., (1987) “Information Systems Failures: A Survey and Classification of the Empirical Literature”, Oxford Surveys in Information Technology Vol. 4, pp. 257-309.

Markus M. and Robey D., (1983) “The Organisational Validity of Management Information Systems”, Human Relations, Vol. 36 No. 3, pp. 203-226.

Miles, M. B., and Huberman, A. M. (1994) Qualitative Data Analysis, Sage Publications, Thousand Oaks, California.

Miller, J. \& Doyle, B., (1987) “Measuring the effectiveness of computer-based information systems in the financial services sector”, MIS Quarterly, Vol. 11 No. 1, pp. 107-124.

N.A.O., (1991) Managing Computer Projects in the National Health Service. HMSO, London.

N.A.O., (1996) The NHS Executive: The Hospital Information Support Systems Initiative. HMSO, London.

Pliskin N., Romm T., Lee, A.\& Weber Y., (1993) “Presumed versus Actual Organisational Culture: Managerial Implications for Implementation of Information Systems”, The Computer Journal, Vol. 36, pp. 143-152.

Rademacher, R. A., (1989) “Critical Factors for Systems Success”, Journal of Systems Management, Vol. 19 (June), 1989, pp. 15-17. 
Reichardt, C. and Cook, T. D. (1989) “Beyond qualitative versus quantitative methods,” In:

Qualitative and Quantitative Methods in Evaluation Research, Reichardt, C. and Cook, T. D., (eds), Sage Publications, Beverly Hills.

Sauer, C., (1993) Why Information Systems Fail: A Case Study Approach, Alfred Waller, Henley.

Stebbins, M., Sena, J. \& Shani, A., (1995) "Information Technology and Organisation Design”, Journal of Information Technology, Vol. 10, pp. 101-113.

Thach, E. and Woodman, R., (1994) "Organisational change and information technology: Managing on the edge of cyber space”, Organisational Dynamics, Vol. 23, 1994, pp. 30-45.

Trend, M. G. (1989) On the Reconciliation of Qualitative and Quantitative Methods in Evaluation Research, Sage Publications, Beverly Hills.

Venkatraman, N., (1991) “IT-induced Business Re-configuration”, In Scott-Morton, M. (ed), The Corporation of the 1990s, Oxford University Press.

Walton, R. E., (1989) Up and Running: Integrating I.T. and the Organisation, Harvard Business School, Boston.

Whyte, G. \& Bytheway, A., (1996) "Factors affecting information systems' success", International Journal of Service Industry Management, Vol. 7 No. 1, pp. 74-93.

Willcocks L. and H. Margetts.,(1994) “Risk Assessment and Information Systems”, European Journal of Information Systems, Vol. 3, No. 2, pp. 127-138.

Wong. E, and G. Tate., (1994) “A Study of User Participation in Information Systems Development”, Journal of Information Technology, Vol. 9, pp. 51-60.

Yap, C., Soh C. and Raman, K., (1992) "Information Systems Success Factors in Small Business”, OMEGA, Vol. 20, No. 5, pp. 597-609. 
Table I: Summary of Success Measures and Scores

Success Measure

Average

Success Score

The CIS in considered to be a technological success in terms of

3.43

accuracy and reliability

The reports produced by the CIS have been relevant, informative and

useful to professional clinical staff

The reports produced by the CIS have been valuable aids to the

decision-making of managers

Professional staff use the CIS regularly to retrieve information, rather

than simply inputting data

Staff like using CIS

Staff are satisfied with the CIS

2.76

The new information provided by the CIS has led to changes in

3.01

decisions, or new decisions by staff

The CIS has enabled practitioners to spend more time providing

direct patient care

The CIS has improved overall job performance

The CIS has allowed clinical staff to be used more efficiently in

2.81

direct patient care 
Table II: Correlation Coefficients Between Best Practice and Success Score

\begin{tabular}{cc}
\hline Statement & Correlation \\
& with Success \\
& Score \\
\hline
\end{tabular}

The CIS was extensively tested by the Trust before it was fully $.4664 * *$ implemented

Extensive support and help was available to staff using the CIS during $\quad .3619 * *$ implementation

The users were actively encouraged to participate in the specification $.3165^{* *}$ of the Trust's requirements in the development of the CIS

The project has active support from top management

A broad training programme exists, designed to address wider issues, . .2612* as well as teaching staff how to use the CIS

The training programme allowed for variations in the skill of the user $\quad .2601^{*}$ population

Enough resources have been allocated to the development and implementation of the CIS

Technical terminology has created difficulties in discussing the system $\quad .0021$ between different groups

Notes: ${ }^{*}$ - Significant at the 0.05 level; ${ }^{* *}$ - Significant at the 0.01 level (2-tailed) 
Table III: Correlation Coefficients for Organisational Impact and Success Score

Statement

Correlation

with Success

Score

The CIS has empowered users by giving greater accessibility to .6565** information in our Trust.

The CIS has had a big impact on the culture of the user groups, where .6303** organisational culture is defined as 'The set of assumptions, beliefs and values, often unstated, that members of an organisation share in common.'

The CIS evoked large changes in users’ clinical working practices in .2963* our Trust.

The CIS has caused large changes in the organisational structure of the $\quad .1051$ Trust.

The CIS evoked large changes in users' non-clinical working practices $\quad-.0751$ in our Trust.

The Trust is having to make large changes in its organisational -.1752 processes to fit with the CIS.

The CIS has not improved the existing flow of information in our $-.3777^{* *}$ Trust.

Clinical staff still keep paper based records to the same extent that $-.5576^{* *}$ they did prior to the CIS.

Notes: * - Significant at the 0.05 level; ${ }^{* *}$ - Significant at the 0.01 level (2-tailed) 